\title{
An exploratory study into the health beliefs and behaviours of British Indians with type II diabetes
}

\author{
Vishal Patel ${ }^{1}$ and Steve Iliffe ${ }^{2}$ \\ ${ }^{1}$ iBSc Medical Student, University College London (UCL), London, England \\ ${ }^{2}$ Professor of Primary Care for Older People, University College London (UCL), London, England
}

\begin{abstract}
Aim: To explore the influence of health beliefs and behaviours on diabetes management in British Indians, as successful management of diabetes is dependent on underlying cultural beliefs and behaviours. Background: British South Asians are six times more likely to suffer from type II diabetes than those in the general population. Yet, little research has been carried out into beliefs about diabetes among the British Indian population. Method: The study used semi-structured interviews, a structured vignette and a pile-sorting exercise. In all, 10 British Indians were interviewed at a General Practice in North West London. Findings: Those interviewed were informed about their diabetes but had difficulties in adapting their diet. Themes identified included causal beliefs of diabetes, use of alternative therapies, moderation of food, adaption of exercise regimes and sources of information. All were aware of avoiding certain foods yet some still continued to consume these items. Participants expressed the need for culturally sensitive forums to help manage their diabetes.
\end{abstract}

Key words: beliefs; diabetes; Indians

Received 5 January 2016; revised 15 June 2016; accepted 4 July 2016; first published online 2 August 2016

\section{Introduction}

In the United Kingdom, there are over 2.5 million individuals diagnosed with diabetes and another half a million more undiagnosed; $90 \%$ suffer from type II diabetes. By 2025, there will be approximately FOUR million people with diabetes, representing a serious challenge to the UK's National Health Service (Khunti et al., 2009).

People of South Asian descent (Indians, Pakistanis and Bangladeshis) are six times more likely to suffer from type II diabetes than those in the general population (Diabetes UK, 2011). The prevalence of type II diabetes among Indian adults is increasing at an even greater rate than the general population, putting them at risk of developing future complications including retinopathy,

Correspondence to: Vishal Patel, 8 Wembley Way, Wembley, Middlesex, HA9 6JJ, UK. Email: vishal.patel.12@ucl.ac.uk

(C) Cambridge University Press 2016 neuropathy, nephropathy and cardiovascular disease (Ramachandran et al., 2010).

Although there is a strong genetic disposition among British Indians to develop defective insulin production and insulin resistance, obesity, lack of physical exercise, and food and eating practices are all contributors. Migrant South Asians in the United Kingdom, United States and around Europe consumed a high calorie and high saturated fat diet and reported lower levels of physical activity than other ethnic groups in those countries which could explain the higher prevalence of diabetes in this group (Garduno-Diaz and Khokhar, 2012).

A qualitative study carried out in a British South Asian population (Patel et al., 2015) emphasised the importance of family influences in diabetes management; for example, eating certain foods such as karella (also known as 'Momordica charanita' or 'bitter melon') was believed to improve diabetes control. Some British Indians 
had other coping mechanisms for their diabetes. In another study carried out in Leeds, UK, 32\% of 106 participants believed their diabetes was 'Allah's will', believing God was the reason behind the cause of their disease (Naeem, 2003).

Culture and religion play a key role in disease within ethnic minorities. References to cultivation and preparation of sugarcane to produce crystallised sugar were first mentioned in the Hindu religious text, the Atharva Veda. Sugar was first discovered in India and traditionally any occasion celebrated by Indians is celebrated with customary offering of sweets to 'sweeten the mouths' of family and friends. During religious festivals it is considered mandatory to offer sweets to the Gods and deities; indicating the cultural and hedonic relevance of sugar to the Indian way of life (Gulati and Misra, 2014).

A study carried out on a British Bangladeshi population found that participants believed that the onset and management of diabetes was dependent on the balance between food entering the body and bodily fluids being secreted (eg, sweat, blood and urine). They believed that the relatively cold weather in England compared with Bangladesh meant an absence of sweat and thus a build up of toxins (Greenhalgh et al., 1998).

There is some pertinent literature regarding the factors that influence management of diabetes in the British South Asian population. However, there is a lack of research into specifically the British Indian ethnic group that this study aims to explore.

\section{Methods}

A mixed methodology study was undertaken using semi-structured interviews and a pile-sorting exercise. Training in interview methods was given before the study commenced and the interviewer (V.P.) discussed interview technique and content with his supervisors. British Indians with type II diabetes registered with a single practice in North London were invited by their general practitioner to take part in interviews about the management of their diabetes with a medical student working in the practice (V.P.).They were provided with an information sheet about the study and invited to express interest. All those who responded positively were interviewed, after written consent had been obtained.

Within the interview, a structured vignette was used to assess participants' knowledge of diabetes (sample section shown in Box 1). The vignettes were only used to inform the interviews and no attempt was made to categorise participants according to their knowledge. Face-to-face interviewing was used as it allowed the use of different data collection techniques. There is evidence to suggest that a conversation style of interviewing produces more accurate data than questionnaires, which was considered as an alternative data collection method (Schober and Conrad, 1997; Krosnick, 1999). Interviews were conducted between March and April 2015. An interview schedule was written with open-ended questions about different aspects of diabetes management. Interviews were audio recorded and transcribed. A thematic analysis approach was taken, led by the interviewer (V.P.) and reviewed by his supervisors. Differences in understanding were resolved through discussion.

Participants were given 10 cards each with a different food item written on them. They were asked to sort the 10 items into two piles: one pile of food which participants thought were healthy for a diabetic and another pile of food which they perceived were bad for a diabetic. Out of the list

Box 1 Sample section of structured vignette

Mr Shah thought if he goes back to the sun and hot temperatures of India, he can sweat it all out and cure his diabetes.

Mr Shah is overweight but he does not think it is linked to his diabetes.

Mr Shah thinks that a 5-min walk to the train station every morning is enough to lose weight.

Primary Health Care Research \& Development 2017; 18: 97-103 
of foods, participants were asked whether they consumed those foods or had cut them out of their diet due to their diabetes. An advantage of using a pile-sorting exercise was that it yielded simple quantitative data which could be expanded upon with the use of the semi-structured interviews to explore any beliefs and behaviours further (Bernard, 1988; 2002).

Interviews lasted between 25 and $50 \mathrm{~min}$. A 'thick description' of participants' behaviours was obtained (Geertz, 1973/1993) and semi-structured interviewing allowed a deeper insight into themes which were arising during the interviews.

\section{Findings}

There were a total of 10 participants and the average age was 61 with eight males and two females. Data analysis yielded five themes: causal beliefs of diabetes, the use of alternative therapies, adaption of exercise, modification of diet and sources of information.

\section{Causal beliefs of diabetes}

Participants highlighted three main causes for diabetes: genetic factors, poor lifestyle choices and stress.

Participants knew about heredity and diabetes:

'I was expecting it because it's like hereditary, both my maternal and paternal grandparents have it'.

$$
\text { (34-year-old woman) }
$$

The majority of participants attributed the cause to their poor diet:

'I think it's because of the type of food we Indians eat. With Indian food, there is a lot of sugar and fat in them. There is a little bit of oil or ghee in mostly everything. It is hard to avoid'.

(63-year-old man)

Some participants described inactivity as a cause of diabetes:

'I think the reason I got diabetes was because during work I was very active and as soon as I retired, I was less active and so I got diabetes'.

(77-year-old man)
A number of participants blamed stress:

'I was under immense stress at work and so to combat that, I found sugars and sweets as a relief'.

(56-year-old man)

\section{Adaptation of exercise}

Exercise was deemed an important aspect of management of diabetes but gyms were not used by any of the participants:

'I don't really have time to be doing any other exercise like going to the gym or what not'.

(56-year-old man)

'Gym is not for me, it's for young people and apart from running on the treadmill or riding the bike, I can't really do any other exercises'.

(58-year-old woman)

Most participants had found ways to increase their levels of physical activity:

'I have a pedometer which tells me I walk about 5 miles a day around the shop floor. I think that is more than enough!'

(56-year-old man)

Others stuck to a day-to-day exercise regime at home and took up hobbies which increased their levels of activity:

'I exercise every day, I wake up every day at 4.15am and do 1 hour treadmill every day. Every day, Monday to Friday. Then Saturday I do gardening, I love my garden. It is a very good hobby for me and it is a good form of exercise too'.

(63-year-old man)

\section{Use of alternative therapies}

A majority of the participants used certain vegetables to control their diabetes. The most common were karella (also known as 'Momordica charanita' or bitter melon) and fenugreek:

'I was watching this program on the TV by a Yoga Swami [Hindu Priest] and he was saying the bitterness of karella is good for diabetics'.

(81-year-old man) 
Some participants admitted to taking alternative medication recommended by family members:

'I take err... Dia-rid... my sister recommended it to me'.

(58-year-old woman)

'When I am feeling a bit different, you know. When I think my sugar is fluctuating too much or I have let myself go a bit with my diet. I guess you can say that I use Dia-rid in a complementary manner along with my, as you call it, allopathic medication'.

(58-year-old woman)

A reluctance to try any alternative medication stemmed from the concern about interactions with their prescribed medication side effects:

'I am happy with my medication at the moment, no side effect, working perfectly so no need to change or experiment. I don't want any possible side effect from any other medication I try'.

(63-year-old man)

Others did not believe in the use of alternative therapies:

'I don't believe in any of it. My cousin is a homeopath in Delhi for 45 years. She keeps telling me to have this or do that. I tell her forget it, you have to have faith in these things, I have no faith in it whatsoever'.

(77-year-old man)

\section{Modification of diet}

Participants all voiced a view that they needed to moderate their food intake:

'Dinners have not changed with standard curry and roti but of course in smaller portions'.

(56-year-old man)

A majority of participants expressed difficulties in changing their diet:

'Your diet depends on where you were brought up and with what types of food. It is very hard to change your diet midway through. From birth, you take up what your parents eat and then maybe you grow up and change a little. Then your children will take that up and change a little. It's a slow transition'.

(77-year-old man)

'Indian traditional sweets, they're so irresistible because they're so nice! So I have had to be more disciplined. We offer sweets to bhagwan [God] and even he does not eat it, he's wise unlike me'.

(56-year-old man)

The pile-sorting exercise showed that participants knew which foods are healthy and not healthy for a diabetic (see Table 1).

\section{Sources of information}

The majority of participants had family or friends who were diabetic and used them to obtain information about the condition and how to

Table 1 Results of the pile-sorting exercise

\begin{tabular}{|c|c|c|c|}
\hline Food item & $\begin{array}{l}\text { Number of participants who } \\
\text { perceive the food item as } \\
\text { healthy for diabetics }\end{array}$ & $\begin{array}{l}\text { Number of participants who } \\
\text { perceive the food item as } \\
\text { unhealthy for diabetics }\end{array}$ & $\begin{array}{l}\text { Number of participants } \\
\text { consuming the food } \\
\text { item }\end{array}$ \\
\hline Ghee & 0 & 10 & 5 \\
\hline Olive oil & 4 & 6 & 2 \\
\hline Beans & 10 & 0 & 10 \\
\hline High-fibre vegetables & 10 & 0 & 10 \\
\hline White sugar & 0 & 10 & 3 \\
\hline Pasta & 6 & 4 & 6 \\
\hline Rice & 6 & 4 & 6 \\
\hline Soft drinks & 0 & 10 & 2 \\
\hline General spicy food & 3 & 7 & 5 \\
\hline Oats and porridge & 10 & 0 & 9 \\
\hline
\end{tabular}


effectively manage it. Social support was also gained from temples (also known as mandirs):

'I have so many [family and friends] who are borderline or are diabetic too so we are always talking to each other about what we are eating and such and sometimes I go for walks with them too. Help each other in balancing the sugar and keeping healthy'.

(58-year-old woman)

'...plus talking to people at the mandir and my elder son because... he studied Biomedical Sciences. He knows quite a bit'.

(77-year-old man)

Participants looked to the literature provided by GPs and pharmacies:

'Whenever I come to the surgery, I see leaflets about diabetes... I pick them up and read them in my free time'.

(63-year-old man)

However, most of this is written in English and some of the participants had difficulty in reading some of the leaflets:

'My English is not the best but if I don't understand anything I ask one of my children to explain to me... I can read Hindi and Gujarati so it would be nice if there were things I could read for myself'.

(58-year-old woman)

There was interest in culturally sensitive forums adapted for British Indians:

'For some of the older people who have preconceptions about it [diabetes] that need to be corrected. Yeah especially if it's like for the Indian community and someone who is Indian who is leading it then they might listen to them'.

(34-year-old woman)

'Within the Asian community [we] need to be more aware of diabetes. I used to go to many seminars organised by Lion's Club and some Swaminarayan Temples... in the past three or four years, I have not heard of any more of these types of events being organised anymore'.

(63-year-old man)

\section{Discussion}

This small study offers insight into how British Indians think about and try to manage their type II diabetes. Although this study demonstrated the cultural significance of foods (Gulati and Misra, 2014), there was less evident fatalism about health (Naeem, 2003), less attraction to alternative medical models (Greenhalgh et al., 1998) and less emphasis on family influences (Patel et al., 2015). These differences may be due to varying socio-economic status and education, or to cohort changes, as some of the literature is relatively dated.

Those interviewed were informed about their diabetes but had difficulty in adapting their diet. Themes identified included causal beliefs of diabetes, use of alternative therapies, moderation of food, adaption of exercise regimes and sources of information. All were aware of the importance of avoiding foods such as ghee and white sugar, yet some still continued to consume these items. Participants expressed the need for culturally sensitive forums to help manage their diabetes.

Participants highlighted three main causes for diabetes: genetic factors, poor lifestyle choices and stress. Most of those interviewed attributed the cause of diabetes to their poor diet. Participants were well educated in what foods were good and bad for management of diabetes. However, some participants seemed to lack motivation to cut out white sugar, ghee and soft drinks from their diet, for example. Reasons for this included their traditional Indian culture and the foods associated with it. Many individuals had been brought up on this diet and found it hard to change.

Most participants in this study were unwilling to try alternative forms of medication as they were afraid of the side effects and combined effects they might have with other medication or because they had no faith or belief in alternative therapies. There was a clear acknowledgement that an increase in physical activity would help management of diabetes. Participants had adapted their lifestyle to include exercise, whether it be walking, running on a treadmill at home or taking up hobbies like gardening.

Whilst participants learned about diabetes management from members of their social network who also had diabetes, this study suggests

Primary Health Care Research \& Development 2017; 18: 97-103 
that there is an unmet need for culturally sensitive forums where British Indians can talk to one another to improve the management of their diabetes. Stone et al. in 2005 carried out a similar small-scale qualitative study in Leicester which found that patients would also be interested in education sessions in their native language. Although Stone's study and ours are both small scale, they both highlight a key issue which is apparent in not one area or population but two different areas of England with differing populations. More rigorous and detailed research needs to be carried out regarding this apparent so that an effective programme could be implemented in the future for diabetic patients.

\section{Strengths and limitations}

This was a small-scale exploratory study of an under-researched topic carried out in North West London. Findings from this study may not be generalisable to other areas of England or to other communities. In future studies it may be useful to sample from different locations such as religious centres or other community centres accessed by British Indians. The pile-sorting exercise was found to be a useful way of opening discussions with participants, but was not designed to categorise participants. Data validation was not carried out due to time constraints.

\section{Conclusion}

Health beliefs and behaviours are not static but always changing. Different populations have different beliefs and behaviours which play an integral role in the management of diseases. An understanding of health beliefs should be used as a start point for culturally sensitive diabetes education. Furthermore, it should be noted that this is not only an issue that needs to be tackled by primary health care but also by public health as well given the importance of social networks and the desire for more sharing of experiences.

\section{Acknowledgements}

The authors would like to thank the participants of this study and the Department of Primary Primary Health Care Research \& Development 2017; 18: 97-103
Care and Population Health, University College London.

\section{Financial Support}

This research received no specific grant from any funding agency, commercial or not-for-profit sectors.

\section{Conflicts of Interest}

None.

\section{Ethical Standards}

Ethical approval for this research was granted by the NHS Research Ethics Committee in February 2015 (REC Reference: 15/NW/0181). Permission was sought from the NHS Trust Research \& Department (R\&D) and NHS R\&D assurance was granted in March 2015 (R\&D Reference: 171109).

\section{References}

Bernard, H.R. 1988: Research methods in cultural anthropology. Beverly Hills, CA: Sage Publications.

Bernard, H.R. 2002: Research methods in anthropology: qualitative and quantitative methods. Walnut Creek; Oxford: AltaMira Press.

Diabetes UK 2011: Diabetes in the UK 2010: key statistics on diabetes. London: Diabetes UK.

Garduno-Diaz, S.D. and Khokhar, S. 2012: Prevalence, risk factors and complications associated with type 2 diabetes in migrant South Asians. Diabetes Metabolism Research and Reviews 28, 6-24.

Geertz, C. 1973/1993. The interpretation of cultures: selected essays. London and Fontana, CA: Basic Books Inc. Publishers.

Greenhalgh, T., Helman, C. and Chowdhury, A.M. 1998: Health beliefs and folk models of diabetes in British Bangladeshis: a qualitative study. BMJ 316, 978-83.

Gulati, S. and Misra, A. 2014: Sugar intake, obesity, and diabetes in India. Nutrients 6, 5955-974.

Khunti, K., Kumar, S. and Brodie, J. 2009: Diabetes UK and South Asian Health Foundation recommendations on diabetes research priorities for British South Asians. London: Diabetes UK.

Krosnick, J.A. 1999: Survey research. Annual Review of Psychology 50, 537-67.

Naeem, A.G. 2003: The role of culture and religion in the management of diabetes: a study of Kashmiri men in Leeds. Journal of the Royal Society for the Promotion of Health 123, 110-16. 
Patel, N.R., Chew-Graham, C., Bundy, C., Kennedy, A., Blickem, C. and Reeves, D. 2015: Illness beliefs and the sociocultural context of diabetes self-management in British South Asians: a mixed methods study. BMC Family Practice $16,58$.

Ramachandran, A, Ronald Ching, W.M. and Snehalatha, C. 2010: Diabetes in Asia. The Lancet 375, 408-18.
Schober, M.F. and Conrad, F.G. 1997: Does conversational interviewing reduce survey measurement error? Public Opinion Quarterly 61, 567-602.

Stone, M., Pound, E., Pancholi, A., Farooqi, A. and Khunti, K. 2005: Empowering patients with diabetes: a qualitative primary care study focusing on South Asians in Leicester, UK. Family Practice 22, 647-52. 